\title{
The variations in trait role, trait conservatism and habitat heterogeneity confound our understanding on community assembly
}

\author{
Yuanbao $\mathrm{Du}^{1}$, Huije Qiao ${ }^{1}$, Zhixin $\mathrm{Wen}^{1}$, and Qisen Yang ${ }^{1}$ \\ ${ }^{1}$ Chinese Academy of Sciences
}

May 6, 2020

\begin{abstract}
Proper conceptual framework of community assembly is critical for understanding biodiversity patterns. In this work, we raised the concepts, 'predominant/ assistant trait', to characterize distinct trait roles in the processes of community organizing. We further incorporated them into previous theoretical understanding. Upon this theoretical basis, we presented a case study with two rodent datasets in the Hengduan Mountains, China. Due to weak phylogenetic signal and extraordinary habitat heterogeneity, we detected most of the rodent communities appeared random at both phylogenetic and morphological facets, which have prevented us to identify the role of morphological attributes and major process structuring rodent communities. We inferred that the variations in trait role, trait conservatism and habitat heterogeneity are major noise confounding our understanding on community assembly. Based on theoretical modification and empirical demonstration, this work has offered a novel and comprehensive perspective to understand the patterns of phylogenetic and trait-based community structure.
\end{abstract}

\section{Introduction}

How and why biodiversity varies along environmental gradient is one of the fundamental issues in ecology and biogeography (Patino et al.2017; Brown 2014; MacArthur \& Wilson 2001). Proper answering this question is meaningful and essential, not only for understanding biodiversity per se, but also for promoting sustainable biodiversity conservation and relevant government-lead policy making. The observed pattern of biodiversity and underlying mechanism have been constantly debated for centuries (Hutchinson 1959; Darwin 1859). Earlier ecologists have paid more attention to the variation of taxonomic composition (i.e., species richness, evenness and variety) (Allenet al. 2002; Whittaker et al. 2001) and proposed many empirical explanatory hypotheses related to contemporary and/or historical environmental variance (i.e., habitat heterogeneity, energy, water-energy balance, area and geometric constrain) (McCain 2007; Evanset al. 2005; Boyce et al. 2003; Hawkins et al.2003; Colwell \& Lees 2000). Definitely, these traditional taxonomic approaches have provided a comprehensive understanding on community assembly. However, subsequent ecologists have realized that taxonomic measurements alone are insufficient to identify mechanistic processes without regard to interspecific ecological and evolutionary information (e.g., differentiations in ecological niche and evolution) (Guittaret al. 2019; Blonder 2018; Martiny et al. 2015; Liuet al. 2013; Cavender-Bares et al. 2009; Swenson \& Enquist 2009; Wiens \& Graham 2005).

Recently raised phylogenetic and trait-based approaches provide a more reasonable perspective in mechanistic understanding of species assembly (Swenson 2013). Based on classical niche theory (Hutchinson 1959) and phylogenetic niche conservatism hypothesis (PNC) (Wiens \& Graham 2005; Pagel 1999), Webb (2000) offered an approach to calculate community structure based on phylogeny. As a follow-up, Webb et al. (2002) later raised a framework of phylogenetic community structure with which species assembly process could be roughly estimated by measuring phylogenetic structure and evaluating trait conservatism. In the last decade, more and more studies have applied the information of phylogeny and functional trait into understanding community assembly process along environmental gradient (Chun \& Lee 2018; Feng et al. 2014; Cianciaruso 
et al. 2012) at various spatial and temporal scales (Maherali \& Klironomos 2007; Cavender-Bares et al. 2006; Swensonet al. 2006). Meanwhile, the framework of community construction has been constantly modified (Kraft \& Ackerly 2010; Kraft et al.2007; Cavender-Bares et al. 2004). The effect of stochastic process, environmental complexity and the effect of negative density-dependence have been taken into account in later frameworks, which enabled it more practical in interpreting species assembly in real and complex ecosystems (Kraft \& Ackerly 2010; Kraft et al.2007). By examining trait similarity on phylogeny and correlation between phylogenetic and trait dispersion, Cavender-Bares et al.(2004) and Losos et al. (2003) have reported that long history of competitive interactions could produce trait convergence on phylogeny. This means that PNC can interpret trait evolution in some taxa, but does not so in any single case (Losos 2008). In other words, ecologists should carefully examine the magnitude of PNC rather than subjectively presuppose its existence in functional attributes (Losos 2008). This has enhanced the worldwide application of phylogenetic and trait-based approaches to study on the potential process driving community assembly.

Although ecologists have made a mass of efforts to open the 'Pandora's Box' of community construction, the mechanism underlying community assembly are still poorly understood. Under prior frameworks of community structure, if relevant functional attribute is phylogenetically conserved on phylogeny, phylogenetic dispersion is expected to be concordant with the trait dispersion of concern (Kraft \& Ackerly 2010; Kraft et al. 2007; Cavender-Bares et al.2004; Webb et al. 2002). But discordant or opposing patterns between phylogenetic relatedness and phylogenetically conserved trait were also reported in flora (Yang et al. 2014; Swenson \& Enquist 2009) and fauna (Du et al. 2017). Generally, most of empirical studies believed that these contradictory patterns were resulting from the low magnitude of phylogenetic signal (Du et al. 2017; Yanget al. 2014). An alternative explanation points to the potential erroneous inferences provided by phylogenetic signal metrics (Swenson \& Enquist 2009).

Kraft and Ackerly (2010) have ever mentioned that some kinds of traits are more sensitive responding to ecological process than the others. However, it appears extremely challenge to quantify how much one functional attribute relates to a certain ecological function. This is because, on one hand, ecological process usually organizes community by acting on multiple phenotypes and their interactions (Miner et al. 2005; Norberg et al. 2001). Under a certain scenario, the functional roles of ecological attributes usually differs from each other and exhibits distinct dispersion. For instance, plant traits related to productivity should be functional clustering in local plant communities, whereas traits related to disturbance and regeneration are expected to be locally over-dispersed (Swenson \& Enquist 2009; Grime 2006; Thompson et al. 1996). On the other hand, nonrandom patterns of functional structure along environmental gradient have implied the functional role of a certain phenotype likely varies across assemblages (Du et al. 2017). It means that the trait dominating species assembly within one habitat might act an assistant role in another habitat, vice versa. These evidences above mentioned have implied that disentangling distinct functional contribution of traits is essential for revealing the truth underlying community assembly.

A novel framework of phylogenetic and trait-based community structure

Well known, community assembly processes act on species through a series of phenotypes (or frequently called functional traits) (Chai et al.2019; Laughlin \& Messier 2015; Pawar 2015). According to distinct contributions of functional attributes in community assembly, we raised the concepts of "predominant trait" and "assistant trait" to refer major or supporting traits mediating assembly process. Combining prior theoretical efforts (Kraft \& Ackerly 2010; Kraft et al. 2007; Cavender-Bares et al. 2004; Webb et al. 2002) and the thought of distinct contributions displayed by traits, we proposed a novel framework of phylogenetic and trait-based structure.

We built this modified framework on four key assumptions. Firstly, following with prior conceptual work, we assumed that phylogenetic relatedness was a comprehensive proxy of interspecific similarity at multiple ecological niches (Swenson 2013). Secondly, we assumed that functional traits were independent with each other in evolutionary and ecological processes. Because the relationship among phenotypes was extremely complicated and taxon-specified, which was beyond the research scope in this work. Thirdly, we assumed that environmental filtering effect, competitive exclusion and stochastic process are three major processes 
driving community assembly. Other processes such as mutualism, host-pathogen interactions, plant-insect interactions and negative density-dependence mentioned in (Cavender-Bares et al. 2009) were not involved in this study, but deserved to be considered in future studies. Lastly, we assumed that phylogenetic signal metrics such as Pagel's lambda (Pagel 1999) and Blomberg's K (Blomberg et al.2003) could accurately infer the phylogenetic conservatism in functional traits. These assumptions above have also clarified the baselines to apply this framework in interpreting the biodiversity pattern.

We simulated ecological processes and community structure using virtual species $\left(\mathrm{A}^{\sim} \mathrm{H}\right)$, phylogeny and traits (phylogenetic conserved traits: T1 and T3; phylogenetic convergent traits: T2 and T4) (Box 1). Two major parameters concerning trait conservatism (phylogenetically conserved or convergent) and trait contribution (predominant or assistant role) were involved in later prediction on phylogenetic and functional dispersion. Given the effects of environmental filtering and competitive exclusion produce opposite imprints on community structure, as a representative, we only simulated the assembly process driven by environmental filtering effect. Following with Kraft and Ackerly (2010), environmental filtering processes were discussed within simple (Fig. 1, scenario A and B) and complex (Fig. 2 scenario C, D and E) habitats, respectively. Besides, we simplified complex habitat with only two microhabitats, which contained independent predominant and assistant traits.

According to the phylogenetic and functional patterns demonstrated in Fig 1 and 2, the modified framework of phylogenetic and trait-based community structure was summarized in Table 1. Generally, in sample habitat, phylogenetic community dispersion was accordant with the functional dispersion of phylogenetically conserved predominant traits, and opposed to phylogenetically convergent predominant traits. While, dispersion comparison between phylogenetic relatedness and assistant traits in simple habitat became more complicated, which highly depended on the degree of phylogenetic conservatism and inter-trait relationship. In complex habitat, due to multi-directional effect across different microhabitats, phylogenetic and functional dispersion appear complicated and unpredictable.

The rodent communities in the Hengduan Mountains (HMs)

Rodents (Rodentia) are characterized as the mammal lineage with high reproductive capacity, rapid and strong environmental adaptive ability (Schenk et al. 2013; Kozak \& Wiens 2010; Jansa et al.2009). As the most diverse lineage in Mammalia, rodents have undergone an extraordinary adaptive radiation throughout the Cenozoic and have spread into almost all natural and artificial terrestrial ecosystems (Fabre et al. 2012). These ecological characteristics have entitled rodents a key role in local and global biomass cycling (Quintero \& Wiens 2013; Schenk et al. 2013; Kozak \& Wiens 2010; Jansa et al. 2009).

Under the extent of modified conceptual framework, we took rodents in the HMs, China, as our study model to present a case study and try to answer two questions. Firstly, we attempted to (1) reveal the process driving the elevational patterns of phylogenetic and morphological structure and their dependence on climatic variables. Secondly, we aimed to (2) identify the importance of trait conservatism, trait role and habitat heterogeneity in driving phylogenetic and trait-based community structure.

\section{Material and Methods}

Study location and datasets

The HMs $\left(97^{\mathrm{O}}-106^{\circ} \mathrm{E}, 21^{\mathrm{O}}-35^{\mathrm{O}} \mathrm{N}\right)$ lies in the southwest of China, covering most part of Yunnan and Sichuan provinces as well as the east part of Tibet. Aiming to detect the role of trait conservatism, functional role of trait as well as habitat complexity in assembling rodent communities, we extracted two rodent datasets based on local sampling survey (local sampling dataset, LSD) and regional records along elevation (regional elevation-range dataset, RED). LSD was extracted from 15 field surveys along local elevational gradient in HMs, including 80 sampling sites containing 45 rodent species (20 genera, 5 families) (Du et al.2017). Six sampling sites in LSD having been conducted within farmland were excluded in Du et al. (2017) to get rid of anthropic impacts on elevational pattern of rodent community structure. In this work, farmland sampling sites were included within LSD, as we also expected to compare patterns and underlying processes with 
those in RED (which contains all typical vegetation types within HMs). Regional elevation-range dataset (RED) were extracted from historical studies, local surveys and museum records within HMs region (Wen et al.2016a; Wen et al. 2016b). As some of historical collections were conducted according to administrative divisions (i.e., province and county), species surviving within non-typical montane habitats (i.e., arid and semi-arid desert) have been recorded within HMs. In order to get rid of this sampling bias in RED, we further conducted data cleaning and excluded species in non-typical habitats at the edges of HMs. The elevational scope in this work ranged from the base of HMs $(<1000 \mathrm{~m})$ to $5000 \mathrm{~m}$. The base zones below $1000 \mathrm{~m}$ were treated as one elevational band, as many historical records below $1000 \mathrm{~m}$ were hard been further divided. Areas above $1000 \mathrm{~m}$ were equally divided into $100-\mathrm{m}$ elevation bands (i.e., $1000 \mathrm{~m}^{\sim} 1100 \mathrm{~m} ; 1100 \mathrm{~m}^{\sim} 1200 \mathrm{~m} .$. . $4900 \mathrm{~m}^{\sim} 5000 \mathrm{~m}$ ). In total, RED contained 94 rodent species (46 genera, 7 families) distributing within 41 elevational bands in HMs. The taxonomy of rodent species followed Wilson and Reeder (2005), except that we recognize Niviventer ling as being distinct from N. confucianus (Du et al. 2017 and their reference).

\section{Phylogeny reconstruction}

Phylogenies of rodents in phylogenetic analyses in this study were reconstructed using published DNA sequences downloaded from GenBank (http://www.ncbi.nlm.nih.gov/genbank/) (last accessed in July, 2018). We inferred the phylogeny using four mitochondrial DNA genes (Cytb, CoI, 12s-rRNA and 16s-rRNA) and three nuclear DNA genes (IRBP, GHR, and RAG1) (Fig. S2 and S3). DNA sequences that used into phylogeny reconstruction could be found in Table S1. Phylogenetic relationships among rodent species were estimated through Bayesian inference (BI) using MrBayes (version 3.2.5) (Ronquist et al. 2012). Parameter settings and other detailed information in phylogenetic reconstructions can be found in Du et al. (2017).

Morphological attributes and phylogenetic signal

In this work, we quantified four major characters representing 'size' (head-body length, hind-foot length, ear length and tail length) and three characters representing 'shape' (tail/head-body ratio, hind-foot/head-body ratio and ear/head-body ratio) into morphological analyses. All morphological attributes were quantified by the mean values of eight (four males and four females) or four (two males and two females) adult individual specimens measuring (Du et al. 2017).

We examined the phylogenetic signal by estimating Pagel's lambda (Pagel 1999) and Blomberg's K (Blomberg et al. 2003). Pagel's lambda is a parameter scaling the interspecific covariances under Brownian evolution and non-sensitive to taxon number (Pagel 1999), whereas Blomberg's K is a scaled ratio trait variation than expected under Brownian motion (Wang \& Clarke 2014; Blomberg et al. 2003). The values of lambda range from zero to one: if lambda $=0$, trait evolution performs independent of the phylogeny; if lambda $=1$, trait evolve following a Brownian motion model (Wang \& Clarke 2014; Pagel 1999). Blomberg's K usually yields positive values $(\mathrm{K}>0)$, and higher $\mathrm{K}$ values indicate stronger phylogenetic signal (Blomberg et al. 2003): if $\mathrm{K}=1$, trait evolves following Brownian motion model; if $\mathrm{K}>1$, interspecific trait similarity is higher than expected under Brownian motion model; if $\mathrm{K}<1$, interspecific trait divergence is higher than expected under a Brownian model (Wang \& Clarke 2014; Blomberg et al. 2003).

Measuring phylogenetic and trait-based structure

In this work, the standardized mean phylogenetic distance (SES.MPD) and its functional analogy, the standardized pairwise trait distance (SES.PW) were applied to quantify phylogenetic and trait-based community structure (Swenson 2014). Using simulation procedure under a null model (independent swap) (Gotelli \& Entsminger 2001), SES.MPD and SES.PW were calculated with the following equation:

$\mathrm{SES}=\left(\right.$ Mean $_{\text {obs- }}$ Mean $\left._{\text {null }}\right) / \mathrm{sd}_{\text {null }}$

where, Mean $_{\text {obs }}$ is the observed MPD or PW within community, Mean null $_{1}$ is the mean value of MPDs or PWs of 999 randomly generated communities, sd $_{\text {null }}$ is the standard deviations of MPDs or PWs of 999 randomized communities. As species data was binary in each community in two datasets, calculation for phylogenetic and trait-based structure were accomplished without abundance weighted (abundance weighted $=$ false). 


\section{Environmental variables}

There is unified opinion among ecologists that patterns of biodiversity across space (i.e., latitudinal or elevational gradient) is as a result of local adaptation to abiotic and biotic variables but not spatial gradient per se. With the aim to detect which variables have lead community assembly, we extracted six climatic variables (annual mean temperature, AMT; temperature seasonality, TS; annual precipitation, AP; precipitation seasonality, PS; net primary productivity, NPP and potential evapotranspiration, PET), which have been frequently reported relating to small mammals biodiversity (Wen et al. 2016b; Stevens \& Gavilanez 2015; Wu et al. 2013).

Lacking in detailed coordinate information for each sampling site and elevational bands, data extraction for each environmental variable was accomplished with local and regional digital elevation model (DEM). We constructed polygon layer for each local or regional elevation band by constraining vertical and horizontal scope. The mean values of environmental variable within sampling site or band polygons were extracted for each assemblage (details to see Du et al. 2017). AMT, TS, AP and PS were all downloaded from Worldclim (http://www.worldclim.org/) (last accessed in July, 2016) (Fick \& Hijmans 2017) at 30 seconds resolution. NPP, and PET were obtained from MODIS products (MOD17A3 and MOD16A3) (Mu et al. 2011; Mu et al. 2007) accessed from LP DAAC (Land Processes Distributed Active Archive Center; https://lpdaac.usgs.gov/) (last accessed in July, 2016). We performed layer mosaic and projection transformation with ENVI (ver. 4.7) (VIS 2011) and ArcMap (ver. 10.0) (ESRI 2010).

\section{Statistical analyses}

Morphological attributes were all log-transformed and centralized before calculating trait-based community structure. Principal component analysis (PCA) was applied for size and shape related morphological attributes, and the first two components were used to calculate morphological dispersion. Standardized phylogenetic and trait-based dispersion (SES.MPD and SES.PW) were determined with two-tailed t-test: if community structure is significantly higher than those in null communities (SES >1.97), community will be defined phylogenetically or functionally dispersed; if community structure is significantly lower than expected (SES $<-1.97)$, community is characterized as phylogenetically or functionally clustered. If community structure is non-significant different from that in null communities $(-1.975<\mathrm{SES}<1.975)$, community is characterized as random (Kembel et al. 2010; Webb et al.2008; Webb et al. 2002; Webb 2000). Underlying process (environmental filtering, interspecific exclusion and stochastic process) and the functional role of traits were estimated according to our revised empirical framework. The elevational patterns of phylogenetic and trait dispersion were determined using polynomial regressions. Best predictive model were selected according to Akaike information criterion (AIC). Besides, we also quantified the elevational pattern of species richness, which is reasonable in estimating environmental fitness and habitat heterogeneity (Brown 2001).

As ecological process and the functional role displayed by trait are habitat-specified, hence, the approach of linear regression model or correlation, to some extent, is more convenient but probably underestimate the dependence between phylogenetic and trait-based community structure. In contrast, pairwise comparison of this kind approach would be more accurate in predicting assembly process and the functional role of a certain trait. In order to obtain comparable results, we applied these two approaches (i.e., correlation analysis and pairwise comparison) in inferring the relationship between phylogenetic and morphological structure. In comparison analysis, if both phylogenetic and trait dispersion showed same status (clustered, random or dispersed), we called it phylogenetic-trait consistence or congruence; or else, we named it phylogenetic-trait inconsistence or incongruence. Pairwise comparison and correlation analysis have been repeatedly conducted between phylogenetic dispersion and morphological dispersion of size and shape related traits.

With the aim to estimate the environmental dependence for phylogenetic and trait dispersion, we conducted forward selection procedure to choose the best climatic predictor(s). Structure equation models (SEM) were applied to estimate the direct and indirect effects of climatic factors on phylogenetic and trait dispersion. Environmental variables were all log-transformed and centralized in best predictor selection and SEMs. All of these calculations were accomplished under R environment (ver. 3.5.1) (Team 2013). phylogenetic signal 
detection was carried out with the package 'phytools' (Revell 2012); PCA analysis, correlation analysis and polynomial regressions were all accomplished with default packages in R (Team 2013); Following the approach in Swenson (2014), phylogenetic and trait-based structure was calculated with package 'picante' (Kembel 2009) and package 'vegan' (Oksanen et al. 2007; Dixon 2003); Forward selection in climatic factors was finished with 'leaps' (Miller 2002) and 'vegan' (Oksanen et al. 2007; Dixon 2003) packages; SEMs were performed with package 'lavaan' (Rosseel 2012).

\section{Results}

Phylogenetic signal

In phylogenetic signal detection, magnitude and significance of phylogenetic signal detection for morphological attributes varied across two datasets. Both $\lambda$ and $K$ indexes for all morphological attributes were below one, some of which were approaching to zero (LSD: $\lambda_{\text {ear }}<0.001, \mathrm{p}>0.999 ; \lambda_{\text {hindfoot-rat }}<0.001, \mathrm{p}>0.999$. RED: $\left.\lambda_{\text {ear-rat }}<0.001, \mathrm{p}>0.999\right)$ (Table S2). In general, phylogenetic signals in regional dataset (RED) were more significant than that in local dataset (LSD) (Table S2).

Pairwise comparison between phylogenetic and trait-based community structure

For 80 local sampling sites in LSD, nearly but more than a half assemblages presented clustered phylogenetic dispersion (SES.MPD $<0)(45,56.25 \%)$; nearly but more than a half assemblages presented functional clustering $(\mathrm{SES} . \mathrm{PW}<0)(46,57.50 \%)$ in size-related trait dispersion; less than a half assemblages exhibited clustering in shape-related trait dispersion $(35,43.75 \%)$. According to two-tailed t-test $(\alpha=0.05)$, most of rodent assemblages in LSD performed phylogenetic or/and functional random (Table S3).

Similar to LSD, nearly but less than a half assemblages in RED exhibited phylogenetic clustering (20, $48.78 \%$ ); more than one third assemblages presented functional clustering in size-related attributes (14, $34.15 \%)$; nearly but more than a half assemblages were functional clustering in shape-related attributes (23, $56.10 \%)$. According to two-tailed t-test $(\alpha=0.05)$ in RED, only one rodent assemblage presented significant clustering on phylogeny (SES.MPD $<-1.975)(1,2.44 \%)$ with the rest all exhibiting phylogenetically random $(-1.975<$ SES.MPD $<1.975)(40,97.56 \%)$; both size and shape related morphological structure exhibited functional random in all rodent assemblages $(-1.975<$ SES.PW $<1.975)(41,100 \%)$ (Table S3).

As most assemblages in LSD and RED were random at phylogenetic and morphological dimensions, there was extremely high degree of phylogenetic-trait congruence in the pairwise comparison (Table S4).

Elevational patterns and environmental explanations

According to the results of polynomial regression, phylogenetic and morphological structure as well as species richness $(\mathrm{SR})$ in LSD all exhibited significant $(\mathrm{p}<0.05)$ linear or binomial pattern along elevational gradient (Fig. 3 and S1; Table S5). In RED, phylogenetic structure (SES.MPD) and morphological structure for size-related morphological attributes $\left(\mathrm{SES}_{\mathrm{P}} \mathrm{PW}_{\text {size }}\right.$ ) exhibited non-linear relationship with elevation, shaperelated morphological structure and SR exhibited significant linear and quadric relationship with elevation (Fig. 3 and S1; Table S5).

According to the results of forward selection, we found that the best predictors varied across datasets and facets of community structure (Table S6). In SEMs, there were positive correlations between phylogenetic and morphological structures in LSD (Fig. 4, a), which were extremely weak in RED (Fig. 4, b).

\section{Discussion}

The novel framework of phylogenetic and trait-based community structure: distinct trait roles

Since Webb et al. (2002) raised the framework of phylogenetic community structure, mechanistic understanding on community assembly has experienced remarkable progress in the last decade. However, phylogenetic approaches highly rely on phylogenetic relatedness being a comprehensive proxy of ecological similarity (Swenson 2013; Losos 2008), which implies the necessity of phylogenetic-trait intergration in revealing mechanism underlying community assembly. 
Distinct functional role and contribution of traits in assembling species has been mentioned in earlier empirical and theoretical studies (Kraft \& Ackerly 2010; Swenson \& Enquist 2009; Grime 2006; Thompsonet al. 1996). Swenson \& Enquist (2009) and Grime (2006) have detected that plant traits related to productivity often appeared clustered in local communities, while disturbance and regeneration related traits displayed locally over-dispersed. Kraft \& Ackerly (2010) have ever predicted that functional traits related to resource use and environmental tolerance were more likely to answer habitat filtering, resource use strategy traits were more sensitive to interspecific competitive exclusion and niche differentiation, physical and chemical defense traits displayed a major role in responding to enemy-mediated negative density dependence. These information together have presented an apparent signal that part of traits are more important (predominant traits) in responding to a certain ecological process, and the others performed relatively less sensitive (assistant traits) response to assemblying process. Absolutely, either predominant or assistant traits are indispensable niche components at both species and community levels. Actually, it appears extraordinarily challenge to decide which functional attributes display a more important role than the others, as they work as an alliance in most of cases (Zukswert \& Prescott 2017; Grassein et al. 2014).

Based on prior understanding on community structure, we rethink over community assembly process by taking account of distinct functional role (predominant and assistant role) of phenotype. Through simulating assembly process in simple habitat, we found that phylogenetic dispersion was accordant with the functional dispersion of conserved predominant trait, and opposed to the structure of convergent predominant trait. This result of predominant traits could also occur in assistant trait, especially when assistant trait presented similar ecological and evolutionary chacteristics (Table 1). But in most instances, the dispersion of assistant traits appeared unpredictable in simple habitat. By comparison, phylogenetic and trait dispersion in complex habitat appear extremely complicated, as community structure is resulting from similar or/and distinct assembly processes acting on conserved or/and convergent functional attributes. Therefore, it seems impossible to predict the functional role of a certain trait in structuring community within a complex habitat. These patterns have also provided two reasonable explanations for the incongruent dispersion between conserved trait and phylgoenetic relatedness (Du et al.2017; Yang et al. 2014; Swenson \& Enquist 2009): the trait might act an assistant role organizing community in simple habitat; or the community is defined upon a heterogeneous habitat.

Pairwise comparison between phylogenetic and trait-based community structure: the variations in trait conservatism and habitat heterogeneity

\section{Trait conservatism}

Admittedly, basic understanding on trait conservatism through phylogenetic signal detection greatly affects our mechanistic interpretations on assembly process (Losos 2008). Even though prior studies have suggested that lambda is not sensitive to the size of species pool (Wang \& Clarke 2014; Blomberg et al. 2003), however, we found both $\mathrm{K}$ and lambda metrics significantly varied across datasets. Besides, most of morphological traits were phylogenetically convergent, especially in LSD. Significant but weak signal $(\mathrm{K}<1, \mathrm{p}<0.05)$ probably results from adaptive convergence to particular environment and indicates labile phylogenetic signal (Blomberg et al. 2003). These results in phylogenetic signal detection is not surprising especially in the most diverse mammalian lineage(Fabre et al. 2012). Environment driven convergent adaptation in functional traits can largely promote coexistence among distant species, efficiently reduce habitat resources caused competitive exclusion among relatives and ultimately facilitate interspecific differentiation in rodents (Cavender-Bares et al. 2009; Cavender-Bares et al. 2004). Absolutely, a single comparison between two species pools might be less comprehensive to infer general conclusion. However, to some sense, these results have provided us an opportunity to rethink about 'what is phylogenetic signal per se' and 'how to understand the degree of PNC by detecting phylogenetic signal'. After all, phylogenetic signal obtained from a certain species pool could not represent the complete evolutionary history for every lineage (Swenson 2013).

\section{Habitat heterogeneity}

By examining phylogenetic signal and community structure, one of our primary aims is to interpret the 
process driving rodent community construction. Under two-tailed t-test, most of phylogenetic and traitbased community structures in two rodent datasets exhibited random dispersion. Considering abiotic and biotic condition within artificially defined rodent community and the elevational pattern of community structure per se, we prefer to believe that multiple interactions between random and deterministic process in complex habitat have produced random rodent community structure.

Extraordinary habitat heterogeneity in HMs has been frequently reported in previous empirical studies (Wen et al. 2016b; Lei et al. 2015; Lei et al. 2014). Besides, there is an indisputable fact that the amount of microhabitats is highly related to local biodiversity (Mehrabi et al. 2014). Within same area, a regional band with 100 meters range along elevational gradient (RED) should contains more microhabitats than that in local sampling transect (LSD). This inference was supported by the pattern of species richness which is highly related to environmental heterogeneity (Brown 2001). We found that rodent species richness of assemblages in RED was much higher than that in LSD. This should be one reason that phylogenetic and functional community structure of almost all assemblages in RED exhibited random dispersion and elevational pattern (except for SES.PW $W_{\text {shape }}$ in RED) (further discussions to see in the following section).

Elevational patterns of phylogenetic and morphological structure at local and regional spatial scales

Resulting from interactions between stochastic and deterministic processes in heterogeneous habitat, phylogenetic and trait-based structure of most assemblages exhibited random dispersion in two datasets. However, significant linear patterns of SES.MPD, SES.PW size $_{\text {, SES.PW }}$ shape in LSD and SES.PW shape $_{\text {in RED have }}$ implied that the relative importance of deterministic processes (i.e., environmental filtering and competitive exclusion) varied along the elevational gradient. We estimate that apparent elevational patterns of community structure in LSD are resulting from significant niche separation among lineages. Early study has mentioned that three families (Muridae, Cricetidae and Sciuridae) in Rodentia have acted key roles in assembling rodent communities in the HMs (Du et al. 2017). Therein, long-tailed murine species have occupied the complete gradient, whereas short-tailed species of Cricetidae mainly distribute at medium and higher elevations. In addition, hylacolous sciurine species mainly survive in broad-leave and coniferous forests ranging from mid-low to mid-high elevations, except for Marmota himalayana surviving in alpine desert steppe.

In contrast, non-significant linear elevational patterns of SES.MPD and SES.PW $W_{\text {size }}$ in RED are possible resulting from higher environmental heterogeneity and enlarged species component. In RED, the horizontal extent of each elevational band approach 9 degrees and 14 degrees at longitudinal and latitudinal directions. Resulting from extraordinarily neighboring topological and climatic heterogeneity in HMs, regional slice with a 100m-elevation range contains enormous subareas and microhabitats, which has harbored mass of rodent species without substantial overlap in distribution. This artificial treatment might slightly influence the pattern of species diversity, but greatly affect phylogenetic and functional diversity pattern, especially the loss or gain of rare species (Mi et al. 2012). To some extent, this offers an interpretation for the consistent patterns of species richness but distinct phylogenetic and morphological community structure patterns in two datasets.

According to best predictive model selection, we have detected that different facets of community structure performed distinct dependence to environmental variables, and the degree of environmental dependence was much lower at regional scale. Obviously, due to higher level of environmental heterogeneity, enlarged species component and less accurate treatment in extracting environmental variables, the interpreting power of climate predictive models deserve to sharply decline.

Conclusions

By combining prior theoretical efforts (Kraft \& Ackerly 2010; Kraftet al. 2007; Cavender-Bares et al. 2004; Webb et al. 2002) and the thought of distinct contributions displayed by traits (predominant and assistant traits), we proposed a novel framework to understand community assembly. Relying on phylogenetic relatedness acts as an aggregate of interspecific niche dissimilarity, the phylogenetic dispersion in simple habitat deserve to be predictable by assessing the functional dispersion of predominant traits. However, phylogenetic and functional dispersion within complex habitat becomes extremely complicated, which highly 
depends on the variations in trait role, trait conservatism and habitat heterogeneity.

With two datasets of rodents in the HMs, we presented a case study to reveal the dominant ecological process assembling community. Resulting from multiple interactions between stochastic and deterministic processes in heterogeneous habitat, most of rodent assemblages exhibited phylogenetic and functional random, which has prevented us to estimate the functional roles of morphological traits. Due to higher level of habitat heterogeneity and enlarged community components, phylogenetic and morphological structure of rodent community at regional scale exhibited random pattern along elevational gradient and performed weak dependence on environmental variables. Despite of a series of inevitable deficiencies, this work has improved our mechanistic understanding on community assembly.

\section{Acknowledgements}

We appreciate Dr. Xiaojuan Liu from the Institute of Botany (CAS) and Dr. Tianlong Cai from the Institute of Zoology (CAS) for their constructive suggestions and kindly help in manuscript revision. We also thank anonymous reviewers for their efforts and kindly suggestion. This research was funded by a grant from the Ministry of Science and Technology of China (2014FY210200).

\section{References}

Allen, A.P., Brown, J.H. \& Gillooly, J.F. (2002). Global biodiversity, biochemical kinetics, and the energeticequivalence rule.Science, 297, 1545-1548.

Blomberg, S.P., Garland, T., Jr. \& Ives, A.R. (2003). Testing for phylogenetic signal in comparative data: behavioral traits are more labile. Evolution, 57, 717-745.

Blonder, B. (2018). Hypervolume concepts in niche-and trait-based ecology. Ecography , 41, 1441-1455.

Boyce, M.S., Mao, J.S., Merrill, E.H., Fortin, D., Turner, M.G., Fryxell, J. et al. (2003). Scale and heterogeneity in habitat selection by elk in Yellowstone National Park. Ecoscience, 10, 421-431.

Brown, J.H. (2001). Mammals on mountainsides: elevational patterns of diversity. Global Ecol Biogeogr , 10, 101-109.

Brown, J.H. (2014). Why are there so many species in the tropics? J Biogeogr , 41, 8-22.

Cavender-Bares, J., Ackerly, D.D., Baum, D.A. \& Bazzaz, F.A. (2004). Phylogenetic overdispersion in Floridian oak communities. Am Nat, 163, 823-843.

Cavender-Bares, J., Kozak, K.H., Fine, P.V. \& Kembel, S.W. (2009). The merging of community ecology and phylogenetic biology. Ecol Lett, 12, 693-715.

Chun, J.H. \& Lee, C.B. (2018). Partitioning the regional and local drivers of phylogenetic and functional diversity along temperate elevational gradients on an East Asian peninsula. Sci Rep , 8, 2853-2853

Colwell, R.K. \& Lees, D.C. (2000). The mid-domain effect: geometric constraints on the geography of species richness. Trends Ecol Evol , 15, 70-76.

Colwell, R.K., Rahbek, C. \& Gotelli, N.J. (2004). The Mid-Domain Effect and Species Richness Patterns: What Have We Learned So Far? The Am Nat, 163, E1-E23.

Darwin, C. (1859). On the origins of species by means of natural selection. London: Murray, 247.

Dixon, P. (2003). VEGAN, a package of R functions for community ecology.J Veg Sci, 14, 927-930.

Du, Y.B., Wen, Z.X., Zhang, J.L., Lv, X., Cheng, J.L., Ge, D.Y. et al . (2017). The roles of environment, space, and phylogeny in determining functional dispersion of rodents (Rodentia) in the Hengduan Mountains, China. Ecol Evol , 7, 10941-10951.

Evans, K.L., Warren, P.H. \& Gaston, K.J. (2005). Species-energy relationships at the macroecological scale: a review of the mechanisms. Biol Rev , 80, 1-25. 
Fabre, P.H., Hautier, L., Dimitrov, D. \& Douzery, E.J. (2012). A glimpse on the pattern of rodent diversification: a phylogenetic approach. BMC Evol Biol , 12, 88.

Fick, S.E. \& Hijmans, R.J. (2017). WorldClim 2: new 1-km spatial resolution climate surfaces for global land areas. Int J Climatol, 37, 4302-4315.

Freeman, B.G., Tobias, J.A. \& Schluter, D. (2019). Behavior influences range limits and patterns of coexistence across an elevational gradient in tropical birds. Ecography , 42, 1832-1840.

Gotelli, N.J. \& Entsminger, G.L. (2001). Swap and fill algorithms in null model analysis: rethinking the knight's tour. Oecologia , 129, 281-291.

Grime, J.P. (2006). Trait convergence and trait divergence in herbaceous plant communities: Mechanisms and consequences. J Veg Sci, 17, 255-260.

Guittar, J., Shade, A. \& Litchman, E. (2019). Trait-based community assembly and succession of the infant gut microbiome. Nat Commun, 10, 512 .

Hawkins, B.A., Field, R., Cornell, H.V., Currie, D.J., Guegan, J.F., Kaufman, D.M. et al . (2003). Energy, water, and broad-scale geographic patterns of species richness. Ecology , 84, 3105-3117.

Helmus, M.R., Bland, T.J., Williams, C.K. \& Ives, A.R. (2007). Phylogenetic measures of biodiversity. Am Nat , 169, E68-83.

Hutchinson, G.E. (1959). Homage To Santa-Rosalia or Why Are There So Many Kinds of Animals. Am Nat , 93, 145-159.

Jansa, S.A., Giarla, T.C. \& Lim, B.K. (2009). The Phylogenetic Position of the Rodent Genus Typhlomys and the Geographic Origin of Muroidea.J Mammal , 90, 1083-1094.

Kembel, S.W. (2009). Disentangling niche and neutral influences on community assembly: assessing the performance of community phylogenetic structure tests. Ecol Lett , 12, 949-960.

Kembel, S.W., Cowan, P.D., Helmus, M.R., Cornwell, W.K., Morlon, H., Ackerly, D.D. et al . (2010). Picante: R tools for integrating phylogenies and ecology. Bioinformatics , 26, 1463-1464.

Kozak, K.H. \& Wiens, J.J. (2010). Accelerated rates of climatic-niche evolution underlie rapid species diversification. Ecol Lett, 13, 1378-1389.

Kraft, N.J., Cornwell, W.K., Webb, C.O. \& Ackerly, D.D. (2007). Trait evolution, community assembly, and the phylogenetic structure of ecological communities. Am Nat, 170, 271-283.

Kraft, N.J.B. \& Ackerly, D.D. (2010). Functional trait and phylogenetic tests of community assembly across spatial scales in an Amazonian forest. Ecol Monogr , 80, 401-422.

Laughlin, D.C. \& Messier, J. (2015). Fitness of multidimensional phenotypes in dynamic adaptive landscapes. Trends Ecol Evol, 30, 487-496.

Lei, F.M., Qu, Y.H., Song, G., Alstrom, P. \& Fjeldsa, J. (2015). The potential drivers in forming avian biodiversity hotspots in the East Himalaya Mountains of Southwest China. Integr Zool , 10, 171-181.

Lei, F.M., Qu, Y.H. \& Song, G. (2014). Species diversification and phylogeographical patterns of birds in response to the uplift of the Qinghai-Tibet Plateau and Quaternary glaciations. Curr Zool, 60, 149-161.

Liu, X.J., Swenson, N.G., Zhang, J.L. \& Ma, K.P. (2013). The environment and space, not phylogeny, determine trait dispersion in a subtropical forest. Func Ecol, 27, 264-272.

Losos, J.B. (2008). Phylogenetic niche conservatism, phylogenetic signal and the relationship between phylogenetic relatedness and ecological similarity among species. Ecol Lett , 11, 995-1003. 
Losos, J.B., Leal, M., Glor, R.E., De Queiroz, K., Hertz, P.E., Rodriguez S. L. et al . (2003). Niche lability in the evolution of a Caribbean lizard community. Nature, 424, 542-545.

MacArthur, R.H. \& Wilson, E.O. (2001). The theory of island biogeography . Princeton university press.

Martiny, J.B., Jones, S.E., Lennon, J.T. \& Martiny, A.C. (2015). Microbiomes in light of traits: A phylogenetic perspective.Science, 350, aac9323.

McCain, C.M. (2007). Area and mammalian elevational diversity.Ecology, 88, 76-86.

Mehrabi, Z., Slade, E.M., Solis, A. \& Mann, D.J. (2014). The importance of microhabitat for biodiversity sampling. PloS One, 9, e114015-e114015.

Miller, A. (2002). Subset selection in regression. Chapman and Hall/CRC.

Miner, B.G., Sultan, S.E., Morgan, S.G., Padilla, D.K. \& Relyea, R.A. (2005). Ecological consequences of phenotypic plasticity. Trends Ecol Evol, 20, 685-692.

Mi, X., Swenson, N.G., Valencia, R., Kress, W.J., Erickson, D., Ren, H. et al. (2012). The Contribution of Rare Species to Community Phylogenetic Diversity across a Global Network of Forest Plots. Am Nat, 180: 1, E17-E30

Mu, Q., Heinsch, F.A., Zhao, M. \& Running, S.W. (2007). Development of a global evapotranspiration algorithm based on MODIS and global meteorology data. Remote Sens Environ, 111, 519-536.

Mu, Q.Z., Zhao, M.S. \& Running, S.W. (2011). Improvements to a MODIS global terrestrial evapotranspiration algorithm. Remote Sens Environ, 115, 1781-1800.

Norberg, J., Swaney, D.P., Dushoff, J., Lin, J., Casagrandi, R. \& Levin, S.A. (2001). Phenotypic diversity and ecosystem functioning in changing environments: a theoretical framework. Proc Natl Acad Sci U S A, 98, 11376-11381.

Oksanen, J., Kindt, R., Legendre, P., O'Hara, B., Stevens, M.H.H., Oksanen, M.J. et al. (2007). The vegan package. Community ecology package, 10, 631-637.

Pagel, M. (1999). Inferring the historical patterns of biological evolution. Nature, 401, 877-884.

Patino, J., Whittaker, R.J., Borges, P.A., Fernández-Palacios, J.M., Ah-Peng, C., Araujo, M.B. et al. (2017). A roadmap for island biology: 50 fundamental questions after 50 years of The Theory of Island Biogeography. J Biogeogr , 44, 963-983.

Pawar, S. (2015). Chapter Eight - The Role of Body Size Variation in Community Assembly. In: Advances in Ecological Research. Academic Press, pp. 201-248.

Quintero, I. \& Wiens, J.J. (2013). Rates of projected climate change dramatically exceed past rates of climatic niche evolution among vertebrate species. Ecol Lett , 16, 1095-1103.

Revell, L.J. (2012). phytools: an R package for phylogenetic comparative biology (and other things). Methods Ecol Evol , 3, 217-223.

Ronquist, F., Teslenko, M., van der Mark, P., Ayres, D.L., Darling, A., Hohna, S. Larget, B. et al . (2012). MrBayes 3.2: efficient Bayesian phylogenetic inference and model choice across a large model space. Syst Biol , 61, 539-542.

Rosseel, Y. (2012). Lavaan: An R package for structural equation modeling and more. Version 0.5-12 (BETA). J Stat Softw , 48, 1-36.

Schenk, J.J., Rowe, K.C. \& Steppan, S.J. (2013). Ecological opportunity and incumbency in the diversification of repeated continental colonizations by muroid rodents. Syst Biol , 62, 837-864. 
Sherman, R., Mullen, R., Haomin, L., Zhendong, F. \& Yi, W. (2008). Spatial patterns of plant diversity and communities in Alpine ecosystems of the Hengduan Mountains, Northwest Yunnan, China. J Plant Ecol, $1,117-136$.

Stevens, R.D. \& Gavilanez, M.M. (2015). Dimensionality of community structure: phylogenetic, morphological and functional perspectives along biodiversity and environmental gradients. Ecography, 38, 861-875.

Swenson, N.G. (2013). The assembly of tropical tree communities the advances and shortcomings of phylogenetic and functional trait analyses.Ecography, 36, 264-276.

Swenson, N.G. (2014). Functional and phylogenetic ecology in $R$. Springer.

Swenson, N.G. \& Enquist, B.J. (2009). Opposing assembly mechanisms in a neotropical dry forest: implications for phylogenetic and functional community ecology. Ecology , 90, 2161-2170.

Team, R.C. (2013). R: A language and environment for statistical computing.

Thompson, K., Hillier, S.H., Grime, J.P., Bossard, C.C. \& Band, S.R. (1996). A functional analysis of a limestone grassland community. J Veg Sci, 7, 371-380.

VIS, I. (2011). ENVI 4.7-The Environment for Visualizing Images.Boulder, Colorado, USA: ITT Visual Information Solutions .

Wang, X. \& Clarke, J.A. (2014). Phylogeny and forelimb disparity in waterbirds. Evolution , 68, 2847-2860.

Webb, C.O. (2000). Exploring the Phylogenetic Structure of Ecological Communities: An Example for Rain Forest Trees. Am Nat , 156, 145-155.

Webb, C.O., Ackerly, D.D. \& Kembel, S.W. (2008). Phylocom: software for the analysis of phylogenetic community structure and trait evolution.Bioinformatics , 24, 2098-2100.

Webb, C.O., Ackerly, D.D., McPeek, M.A. \& Donoghue, M.J. (2002). Phylogenies and community ecology. Annu Rev Ecol Evol S , 33, 475-505.

Wen, Z., Quan, Q., Du, Y., Xia, L., Ge, D. \& Yang, Q. (2016a). Dispersal, niche, and isolation processes jointly explain species turnover patterns of nonvolant small mammals in a large mountainous region of China. Ecol Evol , 6, 946-960.

Wen, Z., Yang, Q., Quan, Q., Xia, L., Ge, D. \& Lv, X. (2016b). Multiscale partitioning of small mammal $\beta$-diversity provides novel insights into the Quaternary faunal history of Qinghai-Tibetan Plateau and Hengduan Mountains. J Biogeogr , 43, 1412-1424.

Whittaker, R.J., Willis, K.J. \& Field, R. (2001). Scale and species richness: towards a general, hierarchical theory of species diversity. $J$ Biogeogr , 28, 453-470.

Wiens, J.J. \& Graham, C.H. (2005). Niche conservatism: Integrating evolution, ecology, and conservation biology. Annu Rev Ecol Evol S , 36, 519-539.

Wilson, D.E. \& Reeder, D.M. (2005). Mammal species of the world: a taxonomic and geographic reference . JHU Press.

Wilson, D.S. (1975). The adequacy of body size as a niche difference.Am Nat , 109, 769-784.

Wu, Y.J., Colwell, R.K., Rahbek, C., Zhang, C.L., Quan, Q., Wang, C.K.et al. (2013). Explaining the species richness of birds along a subtropical elevational gradient in the Hengduan Mountains. J Biogeogr , 40, 2310-2323.

Xing, Y. \& Ree, R.H. (2017). Uplift-driven diversification in the Hengduan Mountains, a temperate biodiversity hotspot. Proc Natl Acad Sci U S A , 114, E3444-E3451. 
Yang, J., Zhang, G.C., Ci, X.Q., Swenson, N.G., Cao, M., Sha, L.Q.et al . (2014). Functional and phylogenetic assembly in a Chinese tropical tree community across size classes, spatial scales and habitats. Func Ecol , 28, 520-529.

Zukswert, J.M. \& Prescott, C.E. (2017). Relationships among leaf functional traits, litter traits, and mass loss during early phases of leaf litter decomposition in 12 woody plant species. Oecologia , 185, 305-316.

\section{Hosted file}

Tables and Figure legends.docx available at https://authorea.com/users/317952/articles/ 447991-the-variations-in-trait-role-trait-conservatism-and-habitat-heterogeneityconfound-our-understanding-on-community-assembly

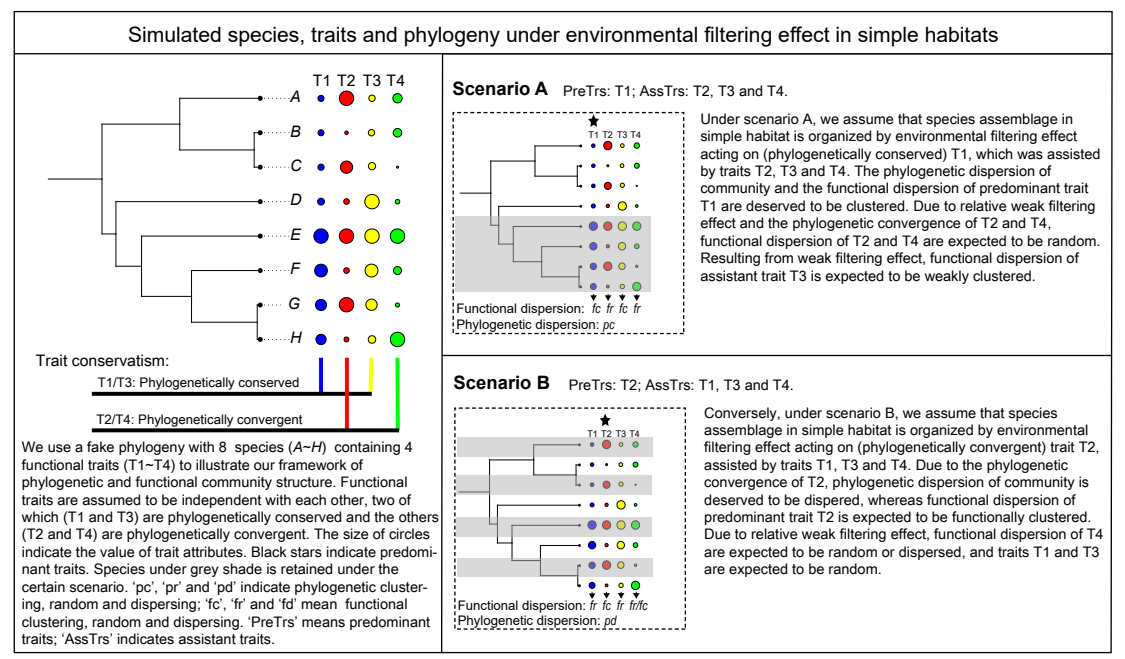




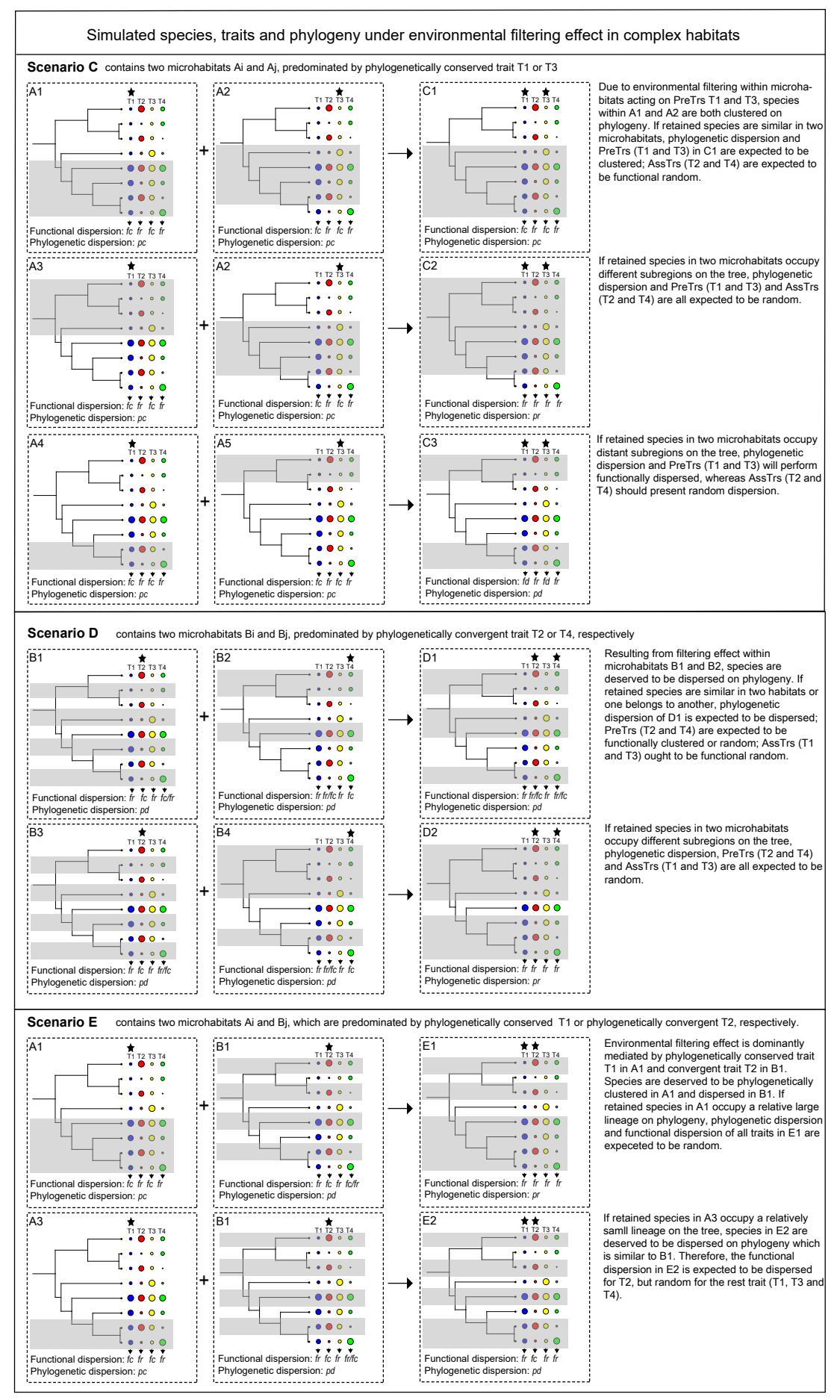



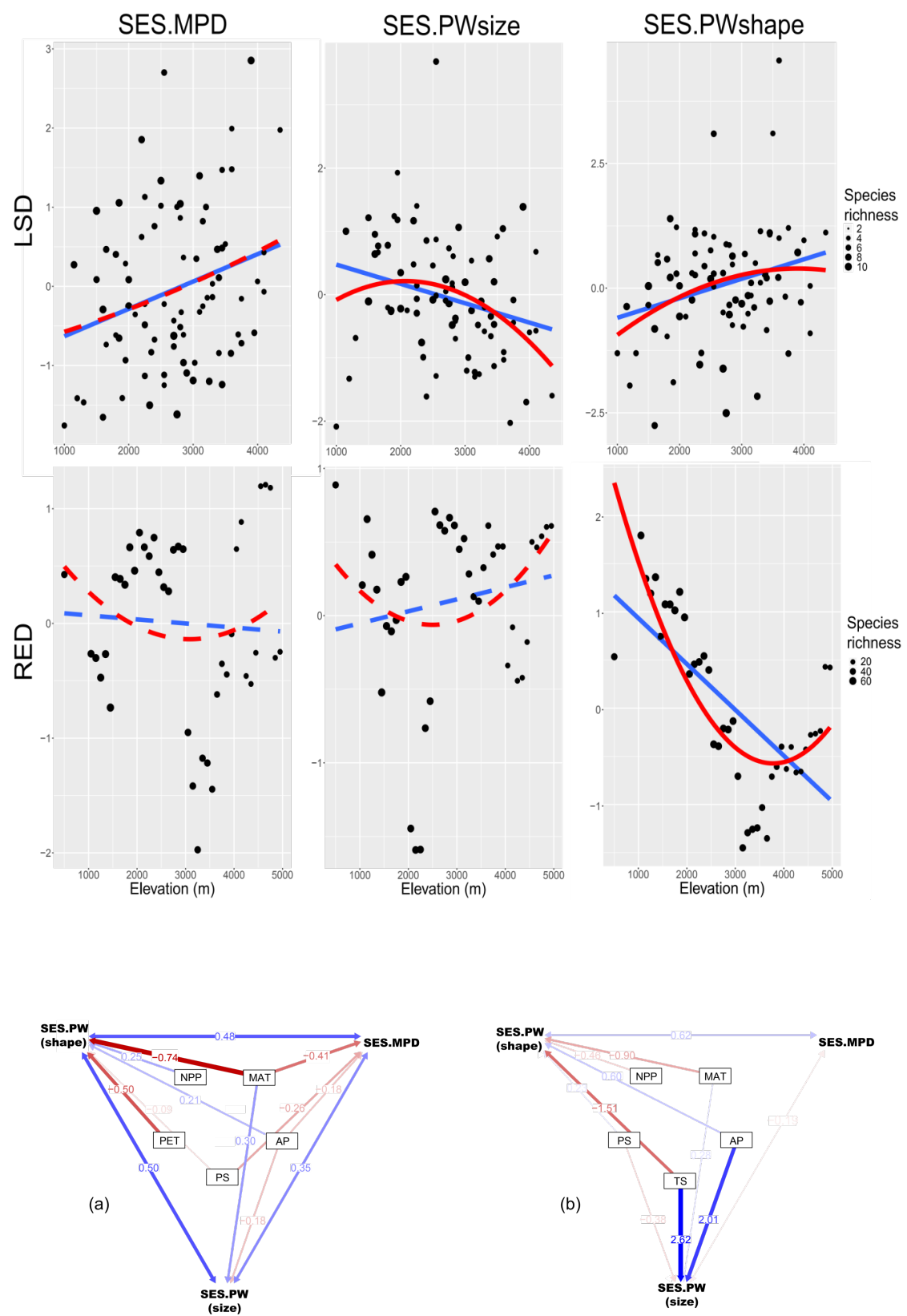